Fabiane Aparecida de Souza Retslaff', Afonso Figueiredo Filho', Andrea Nogueira Dias', Luiz Gastão Bernett ${ }^{2}$, Marco Aurélio Figura ${ }^{2}$

Palavras chave:

Índice de sítio

Curvas h/d

Crescimento e produção

Histórico:

Recebido 23/09/201 I

Aceito $21 / 01 / 2015$

Keywords:

Site Index

Height/DBH curve

Growth and yield

Correspondência: afigfilho@gmail.com

\section{CURVAS DE SÍTIO E RELAÇÕES HIPSOMÉTRICAS PARA Eucalyptus grandis NA REGIÃO DOS CAMPOS GERAIS, PARANÁ}

RESUMO: Neste trabalho, objetivou-se, essencialmente, ajustar modelos para a construção de curvas de índice de sítio e modelos para estimar alturas nas diferentes idades para Eucalyptus grandis, na região dos Campos Gerais, no estado do Paraná. Os dados utilizados no ajuste dos modelos foram provenientes de parcelas permanentes, temporárias e de inventários pré-corte, englobando idades de 2,5 a 26,5 anos. Foram testados vários modelos para representar os sítios e a relação hipsométrica. Para o sítio, o modelo de Chapman-Richards apresentou as melhores estatísticas de ajuste e precisão, tendo-se construído 5 curvas de sítio (amplitude de $5 \mathrm{~m}$ ) pelo método da curva-guia. Os quatro modelos hipsométricos testados apresentaram desempenhos satisfatórios e estatísticas similares, constatando-se que a inclusão da variável altura dominante ou do índice de sítio nos modelos não melhorou, substancialmente, as estatísticas de ajustes e precisão, mas os resíduos ficaram mais homogêneos e mais próximos de zero.

\section{SITE INDEX CURVES AND HYPSOMETRIC RELATIONSHIP FOR Eucalyptus grandis PLANTATIONS FOR THE CAMPOS GERAIS REGION, PARANA STATE}

ABSTRACT: The study aimed to fit mathematical models for the construction of Site Index curves and to estimate heights at different ages for Eucalyptus grandis in the Campos Gerais region, Parana State. The data used to fit the models came from permanent, temporary plots and pre-harvesting inventory, covering ages from 2.5 to 26.5 years. Several models were tested to represent the sites and the hypsometric relationship. The Site Index curves were constructed by the guide-curve method. For the Site Index, the Chapman-Richards model showed the best fit and precision statistics, generating 5 Site Index curves (range of $5 \mathrm{~m}$ ) with the Chapman-Richards model. The four hypsometric models tested showed satisfactory performance and similar statistics and the inclusion of the variables dominant height or site index did not substantially improve the goodness of fit statistics, but the residues were more homogeneous and closer to zero.

' Universidade Estadual do Centro-Oeste - Irati, Paraná, Brasil

2 Klabin AS - Telêmaco Borba, Paraná, Brasil 


\section{INTRODUÇÃO}

Eucalyptus grandis é a espécie de eucalipto mais utilizada em áreas tropicais e de transição para subtropicais no Brasil e em dezenas de outros países, na sua forma pura ou em cruzamentos (PALUDZYSZYN FILHO et al., 2006).

O uso atual de madeira plantada de espécies do gênero Eucalyptus vem recebendo especial atenção, tanto por parte de pesquisadores quanto do setor madeireiro, uma vez que possui um grande potencial de disponibilidade em curto espaço de tempo e ampla diversidade de utilização. A madeira de eucalipto proveniente de plantações, até alguns anos atrás, era voltada exclusivamente para a produção de matériaprima para celulose, carvão vegetal, moirões e postes. Mas, atualmente, com $\circ$ aumento da demanda de mercado por produtos madeiráveis e o crescente desenvolvimento, as empresas do setor florestal estão buscando ampliar as possibilidades de utilização da madeira e diversificar a sua produção (SOARES, 2002).

Em uma empresa florestal, o planejamento da produção é fundamental e, nesse contexto, a modelagem do crescimento e da produção florestal é uma ferramenta que auxilia o manejador na tomada de decisões. A modelagem busca prognosticar a produção em volume, em área basal, ou em peso em função de variáveis que são possíveis de serem quantificadas no povoamento florestal.

Nesse contexto, a modelagem em classes de diâmetro constitui uma ferramenta valiosa para o manejo de plantios florestais, notadamente aqueles destinados para multiprodutos. Esse tipo de modelagem demanda várias ferramentas dendrométricas, dentre elas as curvas de sítio e as curvas hipsométricas têm fundamental importância na prognose da produção.

O crescimento e a produção de um povoamento florestal dependem da idade, da capacidade produtiva ou sítio, do grau de utilização do potencial produtivo e dos tratamentos silviculturais. Assim, a qualidade ou a capacidade produtiva do sítio pode ser definida como o potencial de uma determinada espécie para a produção de madeira (CAMPOS; LEITE, 2009).

Segundo Clutter et al. (1983), no contexto do manejo florestal, a qualidade do sítio pode ser definida como "o potencial de produção de um sítio para uma espécie particular ou um tipo de floresta". As palavras "bom" e "pobre ou ruim" são, frequentemente, utilizadas para classificar a qualidade do sítio e, simplesmente, implicam, respectivamente, em um alto potencial de produção e um baixo potencial. Em razão de grande importância da qualidade do sítio, técnicas têm sido desenvolvidas, métodos para a classificação da qualidade de sítio. Esses métodos podem ser classificados como métodos diretos (estudam o efeito e são os mais empregados) e indiretos (estudam a causa).

A classificação por índices de sítio (método direto) constitui um método prático e consistente da avaliação da qualidade do local, uma vez que todos os fatores ambientais são refletidos de modo interativo no crescimento em altura, a qual também está relacionada com o volume. O fato da classificação do sítio ser feita por meio de uma expressão numérica, em vez de uma descrição qualitativa, constitui-se em uma vantagem desse método (CAMPOS; LEITE, 2009).

As curvas de sítio são construídas a partir de equações de índices de sítio e são utilizadas para classificar a capacidade produtiva do local de modo quantitativo. Essas equações são derivadas de relações funcionais, envolvendo a variável dependente altura dominante média $\left(h_{\text {dom }}\right)$ e como variável independente a idade $(t)$. Elas podem ser construídas, utilizando dados de alturas dominantes e idades advindos de parcelas temporárias, permanentes ou, ainda, de análise de tronco completa (CAMPOS; LEITE, 2009). Exemplos de construção de curvas de sítio para Eucalyptus spp. podem ser encontrados em: Batista e Couto (1986), Dias (2000), Miguel (2009), Nogueira (2003), Retslaff (2010), Santana (2008), Tonini et al. (2006), entre outros.

A altura, assim como o diâmetro, constitui-se em uma importante característica da árvore. Ela pode ser determinada ou estimada, servindo, essencialmente, para - cálculo do volume e para o cálculo de incrementos em altura e em volume. Nos métodos estimativos, a altura entra como segunda variável independente nas tabelas de volume, funções de afilamento e em algumas outras relações dendrométricas (MACHADO; FIGUEIREDO FILHO, 2006).

Em inventários florestais, determinar a altura de todas as árvores pode acarretar mais erros que estimála, em razão das dificuldades de visualização, além de ser uma atividade que demanda tempo e gera custos maiores. A modelagem é uma ferramenta que pode atuar nesse aspecto, uma vez que torna possível o ajuste de equações que estimem a altura de árvores em função do respectivo diâmetro.

Além do diâmetro, outras variáveis como a idade, o índice de sítio ou altura dominante, podem ser utilizadas nos modelos para uma melhor qualidade nos ajustes das curvas de altura. Uma vez que, as curvas 
de h/d (altura/diâmetros ou relação hipsométrica) não apresentam uma relação biológica bem definida e forte, como volume e diâmetro. Os pontos são dispersos em torno da linha média, dificultando identificar modelos que resultem em $R^{2}$ (coeficiente de determinação) altos e erros baixos.

A relação h/d é importante no inventário florestal para a obtenção da estimativa das alturas em uma dada ocasião. Além disso, é importante conhecer essa relação também ao longo da rotação de uma espécie e, nesse aspecto, é de fundamental importância na modelagem do crescimento em classes de diâmetro.

Trabalhos de relações hipsométricas para eucaliptos podem ser encontrados em Zanon et al. (1996), além destes, autores como: Abreu et al. (2002), Dias (2000, 2005), Nogueira (2003), Soares (2002) e Soares et al. (2007), além de utilizarem o DAP (diâmetro à altura do peito) como variável independente para estimar a altura, trabalharam com outras variáveis como $d g$ (diâmetro médio quadrático), $h_{\text {dom }}$ (altura dominante), $t$ (Idade), S (índice de sítio).

Em função da importância das curvas de sítio e das relações hipsométricas para o manejo de plantios florestais, neste trabalho, objetivou-se, principalmente, ajustar modelos para a construção de curvas de índice de sítio e para estimar alturas nas diferentes idades para Eucalyptus grandis na região dos Campos Gerais, estado do Paraná.

\section{MATERIAL E MÉTODOS}

\section{Origem e detalhamento dos dados}

Os dados utilizados, neste estudo, são provenientes de plantios de Eucalyptus grandis localizados no município de Telêmaco Borba, estado do Paraná, disponibilizados pela empresa Klabin S.A. O clima da região, de acordo com o sistema climático de Köeppen, corresponde ao subtropical úmido mesotérmico ( $\mathrm{Cfb}$ ), com verões frescos e geadas severas e frequentes. A temperatura média anual é de $23,0^{\circ} \mathrm{C}$, registrando como temperatura mínima histórica $-5,2^{\circ} \mathrm{C}$ e temperatura máxima histórica de $37,0^{\circ} \mathrm{C}$. A precipitação média anual está em torno de $1.470 \mathrm{~mm}$. A altitude varia de 520 a 1020 metros. A vegetação original pertence ao domínio da Floresta Ombrófila Mista, intermediada pela Estepe Gramíneo-Lenhosa e alguns relictos de Cerrado. Fonte: dados históricos da empresa (FIGURA, 2010).

Os dados são oriundos de 196 parcelas permanentes, 799 parcelas temporárias e 134 parcelas de inventário pré-corte, com área variando de 375 a $900 \mathrm{~m}^{2}$. Em cada parcela, foram medidas todas as circunferências à altura do peito (CAP), as alturas totais das 10 primeiras árvores e das 4 a 9 árvores dominantes, de acordo com a sua área. As idades dos plantios variaram de 2,5 a 26,5 anos.

\section{Desenvolvimento de curvas de sítio}

Existem várias alternativas de construção de curvas de sítio como, por exemplo, o método da curvaguia, o método de atribuição preliminar de índices de sítio, o método da equação das diferenças, e o método de predição de parâmetros. No presente estudo, foi utilizado o método da curva-guia. Esse método gera curvas anamórficas.

Os modelos testados estão apresentados na Tabela I. Para o ajuste foram utilizados I I 29 pares de valores de $h_{\text {dom }}$ e idade $(t)$, com idades variando de 2,5 a 18,6 anos.

TABELA 1 Modelos testados para construção das curvas de Índice de Sítio.

TABLE 1 Tested models for Site Index curves construction.

\begin{tabular}{|c|c|c|c|}
\hline Autor/Fonte & Modelo original & Modelo guia & $\mathrm{N}^{\circ}$ \\
\hline Schumacher & $\mathrm{h}_{\mathrm{dom}}=\mathrm{b}_{0} \cdot \mathrm{e}^{\frac{b_{1}}{t_{i}}} \cdot \mathrm{e}_{\mathrm{i}}$ & $\mathrm{h}_{\mathrm{dom}}=\mathrm{S} \cdot \mathrm{e}^{\beta_{1}\left(\frac{1}{\left.\mathrm{t}_{\mathrm{i}}-\frac{1}{\mathrm{t}_{\text {ref }}}\right)}\right)}$ & [1] \\
\hline Chapman- Richards & $\mathrm{h}_{\mathrm{dom}}=\beta_{0}\left[\left(1-\mathrm{e}^{\left(-\beta_{1} \mathrm{t}_{\mathrm{j}}\right)}\right)\right]^{\beta_{2}} \cdot \varepsilon_{\mathrm{i}}$ & $h_{d o m}=S \cdot\left[\frac{\left(1-e^{\left.\left(-\beta_{1} \cdot t_{i}\right)\right)}\right.}{1-e^{\left(-\beta_{1} \cdot t_{\text {ref }}\right)}}\right]^{\beta_{i}}$ & [2] \\
\hline Mitscherlich & $\mathrm{h}_{\mathrm{dom}}=\beta_{0}-\beta_{1} \cdot \beta_{2}^{\mathrm{t}_{\mathrm{i}}} \cdot \varepsilon_{\mathrm{i}}$ & $h_{\text {dom }}=S-\beta_{1} \cdot\left(\beta_{2} t_{i}-\beta_{2} t_{\text {ret }}\right)$ & [3] \\
\hline Clutter-Jones & $\mathrm{h}_{\mathrm{dom}}=\beta_{0} \cdot\left[1+\beta_{1} \cdot \mathrm{t}_{\mathrm{i}} \beta_{2}\right]^{\beta_{3}} \cdot \varepsilon_{\mathrm{i}}$ & $h_{\text {dom }}=S \cdot\left[\frac{1+\beta_{1} \cdot t_{i} \beta_{2}}{1+\beta_{1} \cdot t_{r e f} \beta_{2}}\right]^{\beta_{3}}$ & [4] \\
\hline
\end{tabular}

$\mathrm{h}_{\text {dom }}=$ altura dominante $(\mathrm{m}) ; \mathrm{S}=$ índice de sítio na idade de referência $(\mathrm{m}) ; \mathrm{t}_{\text {ref }}=$ idade de referência ou idade índice (15 anos); $\mathrm{t}_{\mathrm{i}}=$ idade (anos) da parcela no momento da medição da altura; $\beta_{\mathrm{i}}=$ coeficientes dos modelos a serem estimados; $\varepsilon_{\mathrm{i}}=$ erro aleatório; $\mathrm{N}^{\circ}=$ número do modelo. 
Após a estimativa dos coeficientes dos modelos de índice de sítio, foram geradas as curvas de índice de sítio anamórficas. A idade-índice utilizada foi de 15 anos e a amplitude das curvas foi de $5 \mathrm{~m}$. Foram construídas 05 (cinco) curvas de sítio, definidas em função da distribuição gráfica dos pontos.

\section{Equação hipsométrica}

O ajuste dos modelos hipsométricos foi realizado com dados de altura total, diâmetro, idade, altura dominante e índice de sítio, oriundos do banco de dados de parcelas permanentes, parcelas temporárias e inventário pré-corte. Foram utilizadas 5776 observações para os ajustes dos modelos. Os modelos testados para estimar a altura total estão apresentados na Tabela 2.

Para evitar estratificações e, consequentemente, vários ajustes, o modelo de Prodan (5) foi modificado com a inclusão da variável idade $(t)$, permitindo a obtenção de uma única equação hipsométrica para estimativa da altura total (SANTANA, 2008). Além dos modelos tradicionais de Prodan e Curtis, foram testados dois modelos citados por Nogueira (2003) para estimar a altura total. Nesses dois modelos, uma variável da qualidade do local $\left(h_{\text {dom }}\right.$ ou S) é inserida como variável independente.

Antes do ajuste dos modelos hipsométricos, as parcelas foram classificadas com o Índice de Sítio $(S)$, de acordo com a altura dominante $\left(h_{\text {dom }}\right)$ da parcela na idade de medição.

\section{Análise e seleção dos modelos}

Para análise e seleção de todos os modelos ajustados foram utilizadas as seguintes estatísticas: Índice

TABELA 2 Modelos testados para estimar altura total $(\mathrm{m})$. TABLE 2 Tested models to estimate the total height $(\mathrm{m})$.

\begin{tabular}{cc}
\hline Autor/Fonte & $\mathrm{N}^{\circ}$ \\
\hline $\begin{array}{c}\text { Prodan } \\
\text { modificado } \\
\text { (SANTANA, 2008) }\end{array}$ & $\mathrm{h}-1,3=\frac{\mathrm{d}^{2}}{\beta_{0}+\beta_{1} \mathrm{~d}+\beta_{2} \mathrm{~d}^{2}+\beta_{3} \mathrm{~d} \cdot \mathrm{t}}+\varepsilon_{\mathrm{i}}$ \\
Curtis & $\ln (\mathrm{h})=\beta_{0}+\beta_{1}\left(\frac{1}{\mathrm{~d}}\right)+\beta_{2}\left(\frac{1}{\mathrm{t}}\right)+\beta_{3}\left(\frac{1}{\mathrm{~d} \cdot \mathrm{t}}\right)+\varepsilon_{\mathrm{i}}$
\end{tabular}

$\mathrm{h}=$ Altura total estimada $(\mathrm{m}) ; \mathrm{d}=\operatorname{DAP}(\mathrm{cm}) ; \mathrm{t}=$ Idade do povoamento (anos); $\mathrm{h}_{\text {dom }}=$ altura dominante $(\mathrm{m}) ; \mathrm{S}=$ Índice de Sítio $(\mathrm{m}) ; \beta_{\mathrm{i}}=$ coeficientes de regressão a serem estimados; $\varepsilon_{\mathrm{i}}=$ erro aleatório; $\mathrm{N}^{\circ}=$ número do modelo. de ajuste de Schlaegel $(I A)$, Erro padrão de estimativa $\left(S_{y x} \%\right)$ para a variável de interesse e a análise gráfica da distribuição de resíduos (Res\%).

Na regressão, o nível de significância $\alpha$ adotado foi de $5 \%$ de probabilidade para os coeficientes (ou p-valor $<0,05)$. Segundo Gujarati (2006), o $p$-valor ou valor $p$ (isto é, o valor da probabilidade) é também conhecido como nível de significância exato ou observado, mas, tecnicamente, o valor $p$ é definido como $\circ$ menor nível de significância ao qual a hipótese nula pode ser rejeitada. Quando algum coeficiente não foi significativo, o modelo foi novamente ajustado sem a variável ligada ao coeficiente.

\section{RESULTADOS E DISCUSSÃO}

\section{Curvas de sítio}

$\mathrm{Na}$ Tabela 3, são apresentados os modelos ajustados com as estimativas de seus parâmetros e as estatísticas IA e $S_{y x}$. Pode-se observar que os modelos de Chapman-Richards e o de Mitscherlich apresentaram as melhores estatísticas, seguidos pelos modelos de Schumacher e Clutter-Jones.

Por meio da análise gráfica de resíduos (Figura I) podem-se observar, nas primeiras idades, todos os modelos apresentaram uma tendência em superestimar as alturas.

Para cada modelo testado, foram construídas as curvas de sítio, com amplitude de $5 \mathrm{~m}$. Essa amplitude foi definida em função da distribuição dos valores observados de $h_{\text {dom }} \times$ Idade nas várias idades e na idadeíndice de 15 anos. Assim, foram definidas cinco curvas de sítio para cada modelo e os índices de sítio variaram de 33 a $53 \mathrm{~m}$ na idade-índice de 15 anos. Esse procedimento possibilitou que as curvas englobassem todos os dados e com valores inteiros, de fácil aplicação prática.

$\mathrm{Na}$ Figura 2 podem-se observar as curvas construídas para cada modelo. As curvas geradas pelos modelos de Schumacher e Chapman-Richards se ajustaram bem aos dados, abrangendo a grande maioria desses. As curvas geradas pelo modelo de Mitscherlich foram inadequadas para representar as alturas dominantes nas primeiras idades. O modelo de Clutter-Jones apresentou uma inclinação mais acentuada que os demais modelos, não representando bem o que acontece com as alturas nas maiores idades, que têm tendência assintótica.

Os modelos testados para expressar as curvas de sítio apresentaram boas estatísticas de ajuste e precisão com vantagens para o modelo biológico de 
TABELA 3 Coeficientes e estatísticas (índice de ajuste de Schlaegel - IA e erro padrão de estimativa - Syx) dos modelos testados para construção das curvas de sítio

TABLE 3 Coefficients and statistics (Schlaegel adjustment index - Al and standard error of estimate - Syx) from tested models for the Site Index curves construction

\begin{tabular}{|c|c|c|c|c|c|c|}
\hline Autor/Fonte & Modelos & Coeficientes & IA & Syx (m) & Syx \% & $\mathrm{N}^{\circ}$ \\
\hline Schumacher & $\mathrm{h}_{\mathrm{dom}}=\beta_{0} \cdot \mathrm{e}^{\left(\frac{\beta_{1}}{\mathrm{t}_{\mathrm{i}}}\right)} \cdot \varepsilon_{\mathrm{i}}$ & $\begin{array}{l}\beta_{0}=4,02059 \\
\beta_{1}=-3,40675\end{array}$ & 0,8867 & 3,54 & 9,5 & [1] \\
\hline Chapman-Richards & $h_{d o m}=\beta_{0}\left[\left(1-\exp \left(-\beta_{1} \cdot t\right)\right)\right]^{\beta_{2}} \cdot \varepsilon_{i}$ & $\begin{array}{l}\beta_{0}=57,2934 \\
\beta_{1}=0,09084 \\
\beta_{2}=0,77170\end{array}$ & 0,8999 & 3,33 & 8,9 & [2] \\
\hline Mitscherlich & $\mathrm{h}_{\mathrm{dom}}=\beta_{0}-\beta_{1} \cdot \beta_{2}{ }^{\mathrm{t}} \cdot \varepsilon_{\mathrm{i}}$ & $\begin{array}{l}\beta_{0}=54,9670 \\
\beta_{1}=50,4367 \\
\beta_{2}=0,89380\end{array}$ & 0,8994 & 3,34 & 8,9 & [3] \\
\hline Clutter-Jones & $h_{d o m}=\beta_{0} \cdot\left[1+\beta_{1} \cdot t \beta_{2}\right]^{\beta_{3}} \cdot \varepsilon_{i}$ & $\begin{array}{l}\beta_{0}=5,71539 \\
\beta_{1}=29,3278 \\
\beta_{2}=2,44082 \\
\beta_{3}=0,208367\end{array}$ & 0,8928 & 3,45 & 9,2 & [4] \\
\hline
\end{tabular}

$\mathrm{h}_{\text {dom }}=$ altura dominante $(\mathrm{m}) ; \mathrm{S}=$ índice de sítio $(\mathrm{m})$ na idade índice (15 anos); $\mathrm{t}=$ idade (anos) da parcela no momento da medição da altura; $\beta_{\mathrm{i}}=$ coeficientes estimados; $\mathrm{n}=$ número de observações; $\varepsilon_{\mathrm{i}}=$ erro aleatório; $\mathrm{N}^{\circ}=$ número do modelo.
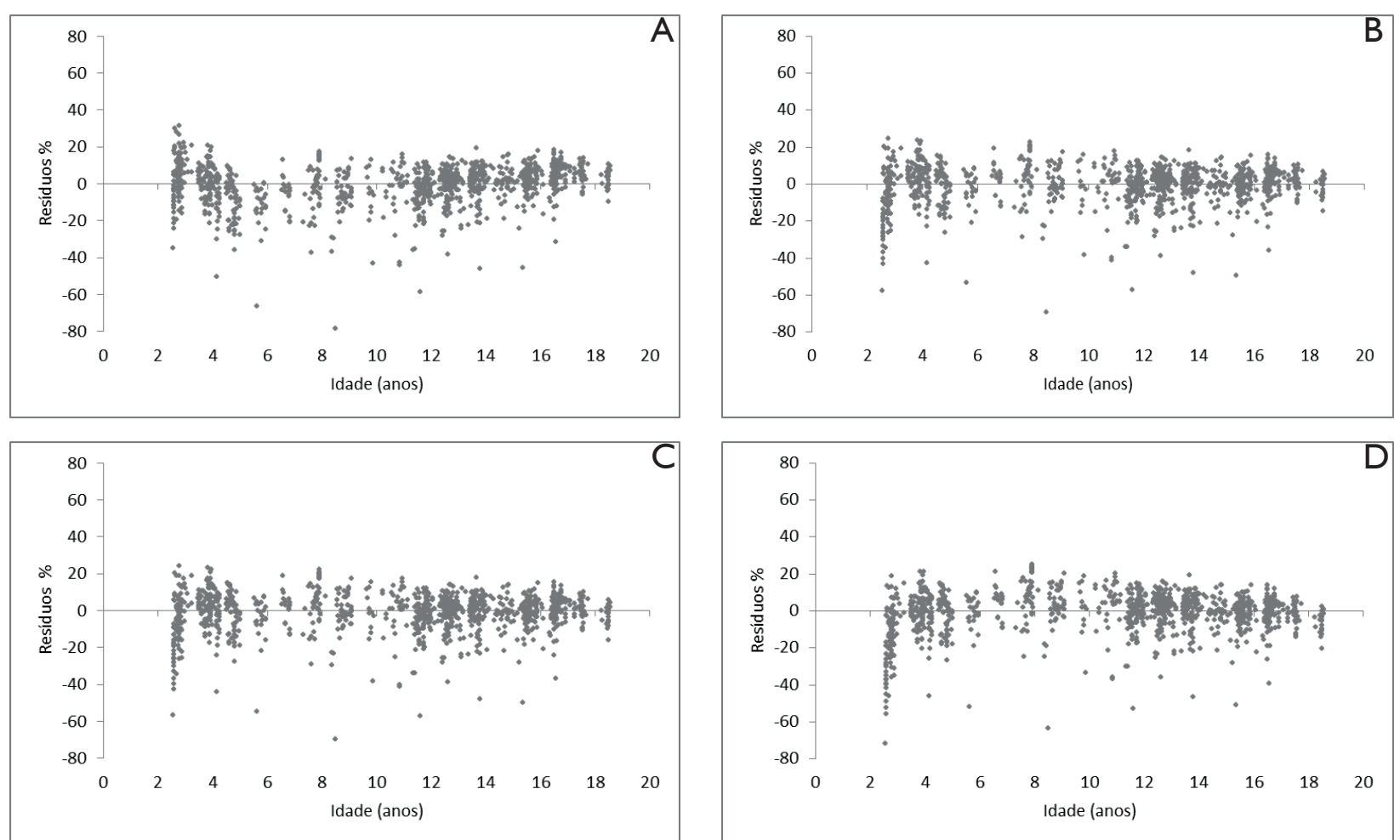

FIGURA 1 Distribuição de resíduos (\%) para a relação hdom x Idade. (A): Schumacher; (B): Chapman-Richards; (C): Mitscherlich; (D): Clutter-Jones

FIGURE 1 Residues distribution (\%) for the relationship hdom x Age. (A): Schumacher; (B): Chapman-Richards; (C): Mitscherlich; (D): Clutter-Jones

Chapman-Richards que apresentou resultados um pouco superiores, além de apresentar características lógicas esperadas em projeções sendo, portanto, o modelo escolhido para estimar os índices de sítio.
O modelo de Chapman-Richards, escolhido para estimar os índices de sítio, também foi selecionado por Santana (2008), para classificar a qualidade produtiva de Eucalyptus grandis na região nordeste do Paraná. 0 


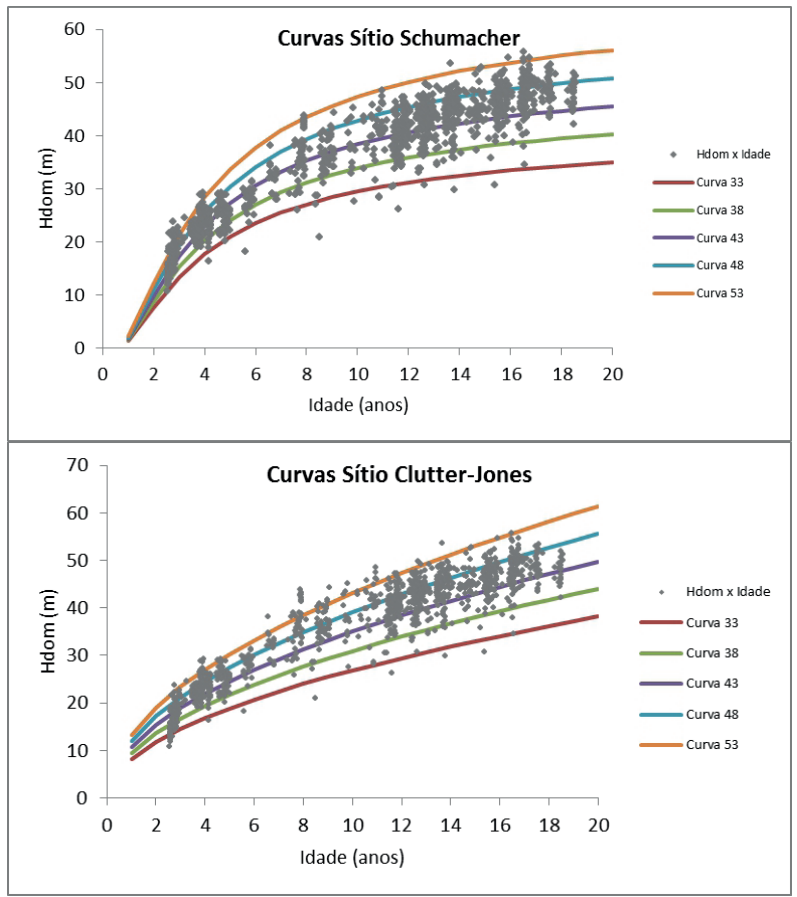

FIGURA 2 Curvas de sítio para os quatro modelos testados. FIGURE 2 Index curves for the four tested models.

autor justificou a escolha, salientando que, além dos bons resultados, o modelo tem também um amplo uso em estudos com espécies de eucalipto no Brasil. Esse modelo também foi selecionado por Miguel (2009) para classificar sítios na região norte do estado de Goiás para povoamentos de Eucalyptus urophylla. Em contrapartida, para Dias (2000) o modelo de Schumacher foi escolhido para classificação da capacidade produtiva para povoamentos de eucaliptos na região nordeste da Bahia.

\section{Equação hipsométrica}

Na Tabela 4, estão os resultados dos ajustes dos modelos para estimar a altura. Os modelos de Prodan (5) e Curtis (6), que estimam a altura em função da idade e do DAP, apresentaram estatísticas semelhantes; ambas as equações têm uma tendência em superestimar as alturas nos menores diâmetros (Figura 3). Os modelos (7) e (8), que envolvem o sítio ou a altura dominante, além das variáveis idade e DAP, apresentaram estatísticas um pouco superiores aos outros dois modelos, porém indicam, também, tendência em superestimar as alturas nos menores diâmetros. A equação resultante do modelo (7) apresentou estatísticas um pouco superiores em relação à equação do modelo (8), a dispersão de resíduos foi similar para as duas equações, no entanto, os resíduos para a equação do modelo (7) estão mais próximos ao
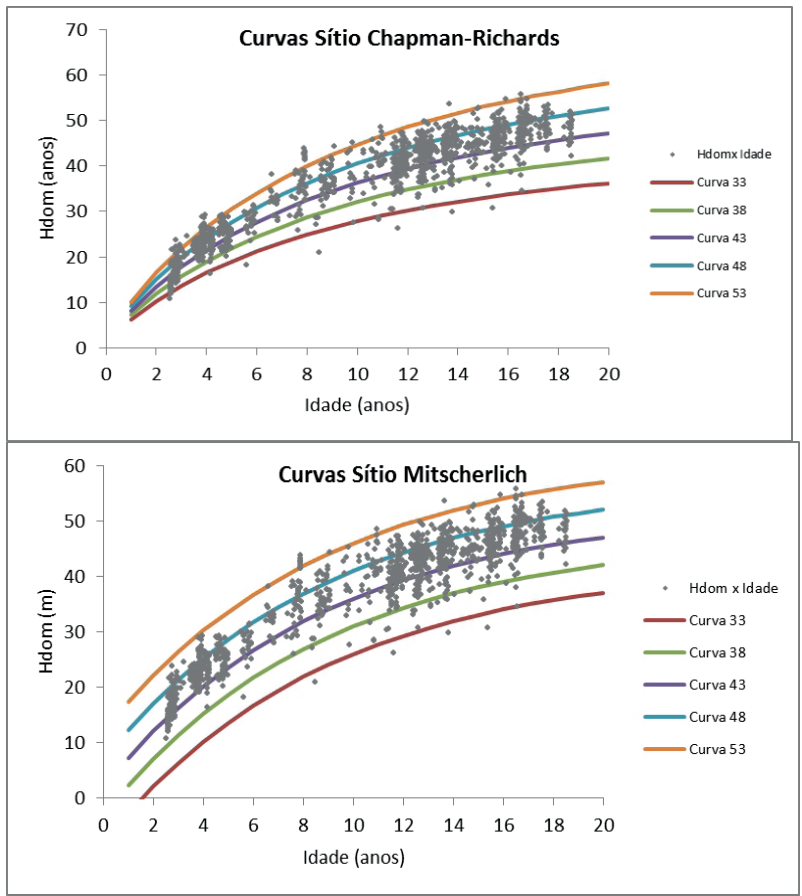

eixo-x que a equação do modelo (8), sendo, portanto escolhida para estimar a altura total.

Zanon et al. (1996), trabalhando com modelos para estimar a altura em função apenas do DAP (relação $\mathrm{h} \times \mathrm{d}$ ) para Eucalyptus dunnii na região da Depressão Central e Encosta Sudeste do Rio Grande do Sul, obtiveram valores de $R^{2}$, variando de 0,8057 a 0,8952 e $\mathrm{S}_{\mathrm{yx}} \%$ entre 2,52 a 8,96 .

Modelos que estimam a altura em povoamentos de eucaliptos, envolvendo as variáveis sítio e altura dominante, além do DAP, foram utilizados por Dias (2000, 2005), Nogueira (2003), Soares (2002) e Soares et al. (2007), os quais obtiveram bons resultados para o ajuste, como os encontrados no presente trabalho para os modelos (7) e (8). Por exemplo, Nogueira (2003) obteve $R^{2}$ de 0,955 para o Modelo (7) e 0,970 para o Modelo (8) para o híbrido Eucalyptus grandis $x$ Eucalyptus urophylla na região nordeste do Estado da Bahia.

\section{CONCLUSÕES}

O modelo biológico de Chapman-Richards apresentou resultados de ajuste superiores aos demais modelos testados, além de possuir características lógicas para projeções do índice de sítio.

As curvas de sítio construídas são adequadas para a classificação de sítios de Eucalyptus grandis, na região dos Campos Gerais, no estado do Paraná. 
Os modelos hipsométricos testados apresentaram desempenhos satisfatórios e estatísticas similares para as equações resultantes. Constatando-se que a inclusão da variável altura dominante ou índice de sítio nos modelos não melhorou substancialmente as estatísticas de ajustes e precisão, no entanto, os resíduos ficaram mais homogêneos e mais próximos de zero com a inclusão dessas variáveis.

TABELA 4 Coeficientes e estatísticas (índice de ajuste de Schlaegel - IA e erro padrão de estimativa - Syx) dos modelos testados para estimar a altura total

TABLE 4 Coefficients and statistics (Schlaegel adjustment index -Al and standard error of estimate - Syx) from tested models to estimate total height

\begin{tabular}{|c|c|c|c|c|c|c|}
\hline Autor / Fonte & Modelo & Coeficientes & IA & Syx (m) & Syx\% & $\mathrm{N}^{\circ}$ \\
\hline $\begin{array}{l}\text { Prodan } \\
\text { modificado }\end{array}$ & $h-1,3=\frac{d^{2}}{\beta_{0}+\beta_{1} d+\beta_{2} d^{2}+\beta_{3} d \cdot t}$ & $\begin{array}{l}\beta_{0}=2,84511 \\
\beta_{1}=0,53586 \\
\beta_{2}=0,01771 \\
\beta_{3}=-0,02668\end{array}$ & 0,9107 & 3,61 & 11,5 & [5] \\
\hline Curtis & $\ln (h)=\beta_{0}+\beta_{1}\left(\frac{1}{d}\right)+\beta_{2}\left(\frac{1}{t}\right)+\beta_{3}\left(\frac{1}{d \cdot t}\right)+\varepsilon_{i}$ & $\begin{array}{l}\beta_{0}=4,25480 \\
\beta_{1}=-12,12640 \\
\beta_{2}=-3,03258 \\
\beta_{3}=18,25661\end{array}$ & 0,9152 & 3,51 & 11,2 & [6] \\
\hline $\begin{array}{l}\text { Nogueira } \\
(2003)\end{array}$ & $\ln (h)+\beta_{0}+\beta_{1} S=\beta_{2}\left(\frac{1}{d}\right)+\beta_{3} \ln (t)+\varepsilon_{i}$ & $\begin{array}{l}\beta_{0}=2,23256 \\
\beta_{1}=0,01657 \\
\beta_{2}=-7,65226 \\
\beta_{3}=0,36155\end{array}$ & 0,9497 & 2,86 & 9,4 & [7] \\
\hline $\begin{array}{l}\text { Nogueira } \\
(2003)\end{array}$ & $\ln (h)+\beta_{0}+\beta_{1} t+\beta_{2} h_{d o m}=\beta_{3}\left(\frac{1}{d}\right)+\varepsilon_{i}$ & $\begin{array}{l}\beta_{0}=2,93275 \\
\beta_{1}=-0,00968 \\
\beta_{2}=0,02585 \\
\beta_{3}=-7,75498\end{array}$ & 0,9467 & 2,95 & 9,7 & [8] \\
\hline
\end{tabular}
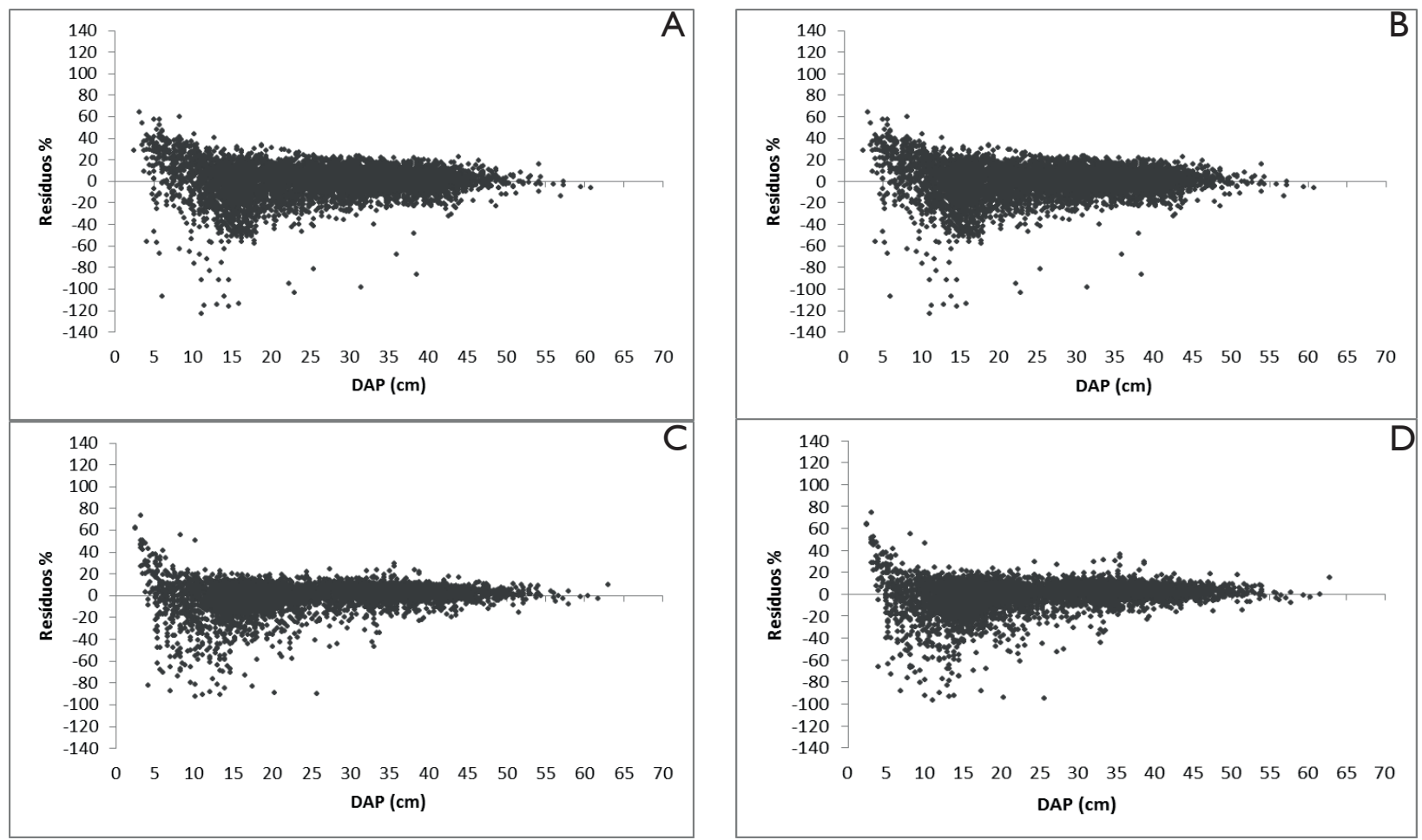

FIGURA 3 Distribuição de resíduos (\%) - (a): Prodan (5); (b): Curtis (6); (c): Modelo (7); (d): Modelo (8).

FIGURE 3 Residues distribution (\%) - (a): Prodan (5); (b): Curtis (6); (c): Model (7); (d): Model (8). 


\section{REFERÊNCIAS}

ABREU, E. C. R.; SCOLFORO, J. R. S.; OLIVEIRA, A. D.; MELLO, J. M.; KANEGAE JÚNIOR, H. Modelagem para prognose precoce do volume por classe diamétrica para Eucalyptus grandis. Scientia Forestalis, Santa Maria, n. 6I, p. 86-I02, jun. 2002.

BATISTA, J. L. F.; COUTO, H. T. Z. Escolha de modelos matemáticos para a construção de curvas de índice de sítio para florestas implantadas de Eucalyptus sp. no Estado de São Paulo. IPEF, Piracicaba, n. 32, p. 33-42, abr. 1986.

CAMPOS, J. C. C.; LEITE, H. G. Mensuração florestal: perguntas e respostas. 3. ed. Viçosa, MG: UFV, 2009.

CLUTTER, J. L.; FORTSON, J. C.; PIENAAR, L. V.; BRISTER, G. H.; BAILEY, R. L. Timber management: a quantitative approach. New York: J. Wiley, 1983.

DIAS, A. N. Modelagem e avaliação econômica de plantações de eucalipto submetidas a desbastes. 2000. 70 p. Dissertação (Mestrado em Ciências Florestais) - Universidade Federal de Viçosa, Viçosa, 2000.

DIAS, A. N. Um modelo para gerenciamento de plantações de eucalipto submetidas a desbaste. 2005. 135 p. Tese (Doutorado em Ciências Florestais) Universidade Federal de Viçosa, Viçosa, 2005.

FIGURA, M. A. A distribuição de weibull na descrição da estrutura diamétrica de Eucalyptus grandis: um enfoque sobre o método dos momentos. 2010. 108 p. Dissertação (Mestrado em Ciências Florestais) Universidade Federal do Paraná, Curitiba, 2010.

GUJARATI, D. N. Econometria básica. Rio de Janeiro: Elsiever, 2006.

MACHADO, S. A.; FIGUEIREDO FILHO, A. Dendrometria. 2. ed. Guarapuava: UNICENTRO, 2006.

MIGUEL, E. P. Avaliação biométrica e prognose da produção de Eucalyptus urophylla (S.T. Blake) na região norte do estado de Goiás. 2009. 165 p. Dissertação (Mestrado em Engenharia Florestal) Universidade Federal do Paraná, Curitiba, 2009.
NOGUEIRA, G. S. Modelagem do crescimento e da produção de povoamentos de Eucalyptus sp. e de Tectona grandis submetidos a desbaste. 2003. I32 p. Tese (Doutorado em Ciência Florestal) - Universidade Federal de Viçosa, Viçosa, 2003.

PALUDZYSZYN FILHO, E.; SANTOS, P. E. T.; FERREIRA, C. A. Eucaliptos indicados para plantio no Estado do Paraná. Colombo: EMBRAPA Florestas, 2006.

RETSLAFF, F. A. S. Modelagem do crescimento e produção em classes de diâmetro para plantio de Eucalyptus grandis. 2010. 168 p. Dissertação (Mestrado em Ciências Florestais) - Universidade Estadual do Centro-Oeste, Irati, 2010.

SANTANA, C. J. de O. Simulação do crescimento e da produção em plantações desbastadas de Eucalyptus grandis com diferentes procedimentos de obtenção dos parâmetros da distribuição Weibull. 2008. 100 p. Dissertação (Mestrado em Engenharia Florestal) Universidade Federal do Paraná, Curitiba, 2008.

SOARES, T. S. Otimização do uso da madeira em povoamentos de eucalipto. 2002. 49 p. Dissertação (Mestrado em Ciência Florestal) - Universidade Federal de Viçosa, Viçosa, 2002.

SOARES, T. S.; LEITE, H. G.; VALE, A. B.; SOARES, C. P.; SILVA, G. F. Avaliação de um modelo de passo invariante na predição da estrutura de um povoamento de Eucalyptus sp. Árvore, Viçosa, v. 3I, n. 2, p. 275-283, 2007.

TONINI, H.; SCHNEIDER, P. R.; FINGER, C. A. G. Curvas de índice de sítio para povoamentos de Eucalyptus saligna Smith para a Depressão Central e Serra do Sudeste, Rio Grande do Sul. Ciência Florestal, Santa Maria, v. 16, n. I, p. 27-43, 2006.

ZANON, M. L. B.; FINGER, C. A. G.; SCHNEIDER, P. R.; KLEIN, J. E. M.; COELHO, M. C. B. Funções para descrever a relação altura diâmetro de Eucalyptus dunnii Maiden. Ciência Rural, Santa Maria, v. 26, n. I, p. 87-90, 1996. 
